Published in final edited form as:

Eur J Radiol. 2018 December ; 109: 68-76. doi:10.1016/j.ejrad.2018.10.014.

\title{
Automated image analysis tool for tumor volume growth rate to guide precision cancer therapy: EGFR-mutant non-small-cell lung cancer as a paradigm
}

\author{
Mizuki Nishino ${ }^{1}$, Satoshi Wakai', Tomoyuki Hida', Suzanne E. Dahlberg ${ }^{3}$, Masahiro Ozaki², \\ Hiroto Hatabu ${ }^{1}$, Hisashi Tachizaki, and Bruce E. Johnson ${ }^{4}$ \\ ${ }^{1}$ Department of Radiology, Brigham and Women's Hospital and Dana-Farber Cancer Institute 450 \\ Brookline Ave. Boston MA, 02215, USA \\ ${ }^{2}$ Canon Medical Systems Corporation 1385 Shimoishigami, Otawara-shi, Tochigi, 324-8550, \\ Japan \\ ${ }^{3}$ Department of Biostatistics Dana-Farber Cancer Institute 450 Brookline Ave. Boston MA, 02215, \\ USA \\ ${ }^{4}$ Department of Medical Oncology and Department of Medicine Dana-Farber Cancer Institute and \\ Brigham and Women's Hospital 450 Brookline Ave. Boston MA, 02215, USA
}

\begin{abstract}
Purpose: To develop an automated analytic module for calculation of tumor growth rate from serial CT scans and to apply the module and evaluate reproducibility in a pilot cohort of advanced NSCLC patients with EGFR mutations treated with EGFR tyrosine kinase inhibitors.

Materials and Methods: The module utilized a commercially available image-processing workstation equipped with a validated tumor volume measurement tool. An automated analytic software module was programmed with the capability to record and display serial tumor volume changes and to calculate tumor volume growth rate over time and added to the workstation. The module was applied to evaluate the tumor growth rate in a pilot cohort of 24 EGFR-mutant patients treated with EGFR inhibitors, and reproducibility was tested by two independent thoracic radiologists.
\end{abstract}

\footnotetext{
Corresponding Author: Mizuki Nishino, MD, MPH, Department of Radiology, Brigham and Women's Hospital and Dana-Farber Cancer Institute, 450 Brookline Ave. Boston MA, 02215, USA, Mizuki_Nishino@DFCI.HARVARD.EDU, Phone: 617-582-7163 Fax: 617-582-8574.

Conflict of Interest:

Nishino: Consultant to Toshiba Medical Systems, WorldCare Clinical, Daiichi Sankyo; Research grant from Merck Investigator Studies Program, Toshiba Medical Systems, AstraZeneca; Honorarium from Bayer, Roche

Dahlberg: Consultancy for AstraZeneca.

Hatabu: Reserch funding from Canon Inc., Toshiba Medical Systems, and Konica-Minolta; Consultant to Toshiba Medical Systems Johnson: Research support: Toshiba Medical Systems and Novartis; Post Marketing Royalties for EGFR Mutation testing; DanaFarber Cancer Institute

Wakai, Tachizaki, Ozaki: Employment: Canon Medical Systems Corporation

Publisher's Disclaimer: This is a PDF file of an unedited manuscript that has been accepted for publication. As a service to our customers we are providing this early version of the manuscript. The manuscript will undergo copyediting, typesetting, and review of the resulting proof before it is published in its final citable form. Please note that during the production process errors may be discovered which could affect the content, and all legal disclaimers that apply to the journal pertain.
} 
Results: The module analyzed chest CT scans from 24 patients (5 males, 19 females; median age: 61 ) with a median of 8 scans per patient, totaling 227 scans and provided a graphical display with an automated and instant calculation of tumor growth rate after the nadir volume for each patient. High inter and intraobserver agreements were noted for tumor growth rates, with concordance correlation coefficients of 0.9323 and 0.9668 , respectively. Interpretation of slow versus fast tumor growth using previously identified threshold of $\$ 0.15 /$ month had a perfect interobserver agreement $(\kappa=1.00)$, and an excellent intraobserver agreement $(\kappa=0.895)$.

Conclusions: The present study describes the development of an image analytic module for assessing tumor growth rate and the data demonstrates the functionality and reproducibility of the module in a pilot cohort of EGFR-mutant NSCLC patients treated with EGFR-TKI. The image analytic module is an initial step for clinical translation of the tumor growth rate approach to guide cancer treatment in precision oncology.

\section{Keywords}

Lung cancer; Epidermal growth factor receptor; EGFR inhibitor; Computed tomography; Tumor growth rate

\section{INTRODUCTION}

Imaging remains a crucial component in the evaluation of precision cancer therapy because it objectively characterizes tumor burden changes, and help to accurately document clinical endpoints ${ }^{1-6}$. Conventionally, RECIST have been widely used as a standard method to evaluate tumor response and define trial endpoints ${ }^{2,3,5}$. However, the limitations of RECIST are increasingly recognized, including a limited reproducibility and a lack of tumor volume burden assessment. CT tumor volume measurements using segmentation tools have been consistently shown to be more reproducible than diameter measurements used in RECIST $^{7-10}$, and reflect the entire tumor volume rather than planar measurements on a selected image. Additionally, RECIST often has limited utility as a guide for treatment decisions. This limitation is most evident in patients treated with precision therapy targeting specific genomic mutations, as represented by non-small-cell lung cancer (NSCLC) patients with sensitizing epidermal growth factor receptor (EGFR) mutations treated with EGFR tyrosine kinase inhibitors (TKIs) ${ }^{10,11}$. EGFR-mutant NSCLC patients respond dramatically to EGFR-TKIs, with response rates of 55-83\% and progression-free survival of 9.7-18.9 months ${ }^{12-17}$. However, their tumors eventually grow back due to acquired resistance and patients experience disease progression ${ }^{18,19}$.

Experienced oncologists often continue EGFR-TKI beyond RECIST progression in EGFRmutant NSCLC patients, because their tumors continue to grow slowly ${ }^{20,21}$. In a phase 2 trial, continuation of erlotinib beyond RECIST progression in EGFR-mutant patients added a median of 3.1 months of treatment period ${ }^{20}$. In another study, $88 \%$ of 56 EGFR-mutant patients treated with EGFR-TKIs continued TKI beyond RECIST progression, indicating that RECIST progression is not a single determining factor to discontinue $\mathrm{TKI}^{6}$. These studies demonstrated the unmet clinical needs for more objective imaging guides to identify patients who can continue EGFR-TKI beyond progression. Similar needs are noted in the setting of other agents such as ALK inhibitors ${ }^{5,22-26}$. 
Important insights for therapeutic decision making can be gained by assessing tumor burden changes over time, or tumor growth rate during therapy ${ }^{27-32}$, which is not currently included in RECIST ${ }^{30,33}$. Several previous reports have evaluated tumor volume growth rate as a marker for defining therapeutic endpoints and assessing clinical benefits ${ }^{28,30-33}$. In our previous report on the volumetric tumor growth rate in 58 EGFR-mutant NSCLC patients after initial response to EGFR-TKI, the overall growth rate of the group after they reached the volume nadir was $0.12 /$ month, for the logarithm of the volume $\left(\log _{\mathrm{e}} \mathrm{V}\right)(\mathrm{SE}: 0.015 /$ month $)^{30}$. The results were reproduced in an independent cohort of 50 EGFR-mutant patients ${ }^{33}$, demonstrating the consistency of the tumor growth rate after nadir in these patients. Given these results, we have proposed an upper limit of $0.15 /$ month for $\log _{\mathrm{e}} \mathrm{V}$ as a threshold to define slow progression for EGFR-mutant patients receiving EGFR-TKI ${ }^{30,33}$, which may provide a more objective tool to help to guide treatment decisions beyond RECIST progression.

While the approach using tumor growth rate analysis has shown promising utility in treatment monitoring and decisions ${ }^{27,28,30-33}$, there are technical barriers to be addressed before the approach can be effectively delivered in the clinical setting and widely used for patient care. Tumor growth rate analyses require recording of serial tumor volume measures at multiple time points throughout therapy, and fitting the measures into a statistical model. Manual procedures for such process are cumbersome and time consuming, and thus are not easily translated into clinical practice.

The purpose of the present study is to develop an analytic module to calculate tumor growth rate using serial quantitative $\mathrm{CT}$ measures for instant interpretation of the results to support clinical treatment decisions, apply the module in a pilot cohort of EGFR-mutant patients and test the reproducibility, for potential deployment of the approach in clinical settings of precision oncology.

\section{MATERIALS AND METHODS}

\section{Overview}

The project utilized a commercially available, technically validated tumor volume measurement tool available on the image-processing workstation (Vitrea, Vital Images) that has been used in the prior studies for tumor growth rate in EGFR-mutant NSCLC.8,30,33,34 Automated analytic software module was developed by an experienced programmer (S.W.) and was added to the Vitrea workstation, to enable automated calculations of tumor growth rate during therapy using the serial tumor volume measures. The module was then applied in a pilot cohort of EGFR-mutant patients treated with EGFR-TKI to test the performance, and reproducibility of tumor growth rate analysis was evaluated with the approval from the institutional review board.

\section{Development of the analytic module for tumor growth rate}

Longitudinal tumor volume tracking-Tumor growth rate analyses utilize the serial tumor volume measurements at all time points from the initiation of therapy, and thus the first function of a new analytic software module consists of capability to record the serial 
tumor volume measurements over time during therapy in each patient. For the purpose of this study, the module was designed to be added to an existing Vitrea workstation equipped with the validated tumor segmentation algorithms that have been used for advanced NSCLC as published before ${ }^{8,18,30,34,35}$. The workflow for tumor volume measurements in advanced NSCLC has also been previously published ${ }^{8,18,30,34,35}$. In brief, axial chest CT images were loaded and displayed on the workstation equipped with tumor volume segmentation software. The reader manually selected the dominant lung lesion by a mouse click to automatically segment the lesion from the surrounding structures using a three-dimensional seed-growing algorithm. The reader then visually evaluated the boundary of the segmented lesion and manually adjusted the boundary of the lesion, which was necessary for all cases of advanced NSCLC, as previously demonstrated. ${ }^{8}$ After segmentation and manual correction, tumor volume of the segmented lesion was automatically calculated. The workstation is DICOM-compatible and can be connected seamlessly with the CT scanners and PACS used in the clinical imaging studies of cancer patients at our institution, and universally accepts DICOM-based CT images regardless of the types of CT scanners used to acquire images (Fig. 1).

The module utilized the advantage of DICOM-based system which allows for the exchange of image-related categorical and quantitative information using DICOM Structured Report (SR) infrastructure, a self-describing information structure that has been employed for the encoding and interchange of clinical trial results derived from imaging studies ${ }^{36}$. The module utilized DICOM SR infrastructure that is designed to enable encoding and storing of tumor segmentation and volume measurement data in a longitudinal fashion. The method allows the module to maintain the detailed records of the patients and their imaging data sets and analysis results, and other relevant information that is archived using DICOM SR infrastructure. As the tumor growth rate analysis requires serial CT scans at multiple time points during therapy, dates and time point identification codes were also created on DICOM SR infrastructure ${ }^{36}$. The structured report allows querying of the imaging and analysis results, enabling the module automatically retrieve the prior studies and results of a corresponding patient.

\section{Graphic display of serial tumor volume dynamics}

The serial records of tumor volume analyses are visually displayed as a trend graph of the longitudinal changes of tumor volume measured at the baseline and on follow-up scans during therapy. The module identifies the initial tumor volume and the nadir volume by comparing all the volume measurements from the initial scan up to the last CT scan obtained from the patient. The date of the scan showing the nadir volume is noted as the nadir time point. In cases where tumor volume does not decrease and keeps increasing since baseline, the baseline volume is recorded as "nadir", per definition ${ }^{30}$. Both the trend graph and nadir are updated each time a measurement is performed. Users are given options for the graph display, using 1) actual volume, 2) a ratio in comparison with the baseline (indicating the baseline volume as "1.0 (100\%)"), and 3) natural logarithm of the actual volume $\left(\log _{\mathrm{e}} \mathrm{V}\right)$ originally measured in $\mathrm{mm}^{3}$. Selection of display options are provided in a drop-down menu, to meet varying needs and preference of the end users. 


\section{Calculation of tumor growth rate and interpretation:}

The module has a function to automatically calculate the tumor volume growth rate over time from the nadir. The calculation of tumor growth rate is performed using the natural $\log$ arithm $\left(\log _{\mathrm{e}} \mathrm{V}\right)$ of the tumor volume originally measured in $\mathrm{mm}^{3}$, as described in prior studies ${ }^{30,37-40}$. For a given patient, the most recent scan can be studied to determine if rapid tumor growth has taken place as defined in the publication on the tumor growth rate in the EGFR-mutant patients, in order to establish "fast" or "slow" tumor growth. The categorization of the growth rate as fast or slow will likely facilitate clinical decisions regarding the patient's therapy. Therefore, the module computes the rate of change in $\log _{\mathrm{e}} \mathrm{V}$ by obtaining the line of best fit for all volume measurements from the nadir during therapy, using the least square method. At a timepoint $t_{i}, x_{i}$ denotes time in months from baseline scan and $V_{i}$ denotes tumor volume measured in $\mathrm{mm}^{3}$. A time point for the minimum value for $\mathrm{V}\left(\mathrm{V}_{\mathrm{p}}\right)$ since baseline is identified as a nadir time point $\left(\mathrm{t}_{\mathrm{p}}\right)$. A sequence of pairs of the time and volume measurements from nadir $\left(\mathrm{t}_{\mathrm{p}}\right)$ to a timepoint $\left(\mathrm{t}_{\mathrm{n}}\right)$ after nadir during therapy is provided: $\left(\mathrm{x}_{\mathrm{p}}, \mathrm{V}_{\mathrm{p}}\right),\left(\mathrm{x}_{\mathrm{p}+1}, \mathrm{~V}_{\mathrm{p}+1}\right),\left(\mathrm{x}_{\mathrm{p}+2}, \mathrm{~V}_{\mathrm{p}+2}\right), \ldots \ldots\left(\mathrm{x}_{\mathrm{n}}, \mathrm{V}_{\mathrm{n}}\right)$; where $\min \left(\mathrm{V}_{\mathrm{p}}, \mathrm{V}_{\mathrm{p}+1}, \ldots . \mathrm{V}_{\mathrm{n}}\right)$ $=\mathrm{V}_{\mathrm{p}}\left(\mathrm{V}_{\mathrm{p}}=\right.$ nadir volume $)$. The volume measured in $\mathrm{mm}^{3}\left(\mathrm{~V}_{\mathrm{i}}\right)$ is converted to the natural $\log$ for the calculation as: $\mathrm{y}_{\mathrm{i}}=\log _{\mathrm{e}} \mathrm{V}_{\mathrm{i}}$. The module calculates the mean of time $(\bar{X})$ and the natural $\log$ of the volume $(\bar{Y})$ for all timepoints since the nadir, and then automatically calculates the slope of the best fit line, $R$, which represents the tumor growth rate for $\log _{\mathrm{e}} \mathrm{V}$ per month at $t_{n}$.

$$
\begin{aligned}
& \bar{X}=\frac{\sum_{i=p^{x}}^{n} x_{i}}{\mathrm{n}-\mathrm{p}+1}=\frac{\sum_{i=p^{y}}^{n} y_{i}}{\mathrm{n}-\mathrm{p}+1}=\frac{\sum_{i=p}^{n} \log V_{i}}{\mathrm{n}-\mathrm{p}+1} \\
& R=\frac{\sum_{i=p^{x_{i}} y_{i}-(\mathrm{n}-\mathrm{p}+1) \bar{X} \bar{Y}}^{n}}{\sum_{i=p^{x}}^{n} x_{i}^{2}-(\mathrm{n}-\mathrm{p}+1) \bar{X}^{2}}
\end{aligned}
$$

The detection of the nadir and the analysis of tumor growth rate after the nadir can be performed every time as a scan is generated during clinical follow-up and the growth rate is recalculated and updated using all the measurements from the nadir at each follow-up, to provide the growth rate at each follow-up scan.

Volumetric tumor growth rate obtained in each patient with EGFR-mutant patients treated with EGFR-TKI can interpreted in reference to the previously published value and its $95 \%$ CIs. The growth rate threshold of $₫ \mathbf{1 5}$ /month has been proposed as the rate that can be interpreted as "slow growth", indicating the patient may safely remain on EGFR-TKI while the tumor are growing back. In contrast, a rate of $>0.15 /$ month will be interpreted as "fast growth", indicating that it is time to consider alternate or additional therapy.

\section{Performance assessment and reproducibility testing in a pilot cohort}

The analytic module for tumor growth rate was added to the Vitrea workstation and was tested in a pilot cohort of 24 EGFR-mutant NSCLC patients (5 males, 19 females; median age: 61 years old) treated with EGFR-TKI and experienced tumor regrowth after an initial response to therapy. All patients had a dominant, solid measurable lesion of the lung ( $\geq 10$ 
$\mathrm{mm}$ ) for tumor volume measurements. Twenty-one patients were treated with erlotinib, and 3 were treated with gefitinib. The median number of CT scans per patient was 8 scans (range: 3-26 scans), totaling 227 scans assessed for tumor volume and analyzed in the module.

Two readers (MN, TH) with expertise in lung cancer imaging independently used the module to obtain tumor growth rate of the dominant lung lesion in the pilot cohort, to assess interobserver variability of the growth rate evaluations. The first reader (MN) repeated the measurements twice, 8 weeks apart between two sets of measurements, to assess intraobserver variability.

The concordance between the tumor growth rate obtained by the readers was assessed using concordance correlation coefficients (CCCs) as published before ${ }^{7,8,34}$. Assuming two assessment results have mean $\mathrm{u}_{1}$ and $\mathrm{u}_{2}$, with variance $\sigma_{1}^{2}, \sigma_{2}^{2}$, and covariance $\sigma_{12}, \mathrm{CCC}=$ $\left(2 \sigma_{12}\right) /\left[\sigma_{1}{ }^{2}+\sigma_{2}^{2}+\left(u_{1-} u_{2}\right)^{2}\right]$. CCCs are composed of a measure of precision (how far each pair of measurements deviates from the best-fit line through the data) and a measure of accuracy (the distance between the best-fit line and the 45 line through the origin). A value of 1 indicates perfect agreement and -1 indicates perfect reversed agreement. Tumor growth rates from two independent readers were used to assess interobserver variability, and the rates from two sets of measurements by the first reader were used to assess intraobserver variability.

\section{RESULTS}

\section{Tumor volume growth rate analysis in the pilot cohort}

The module was able to analyze serial CT tumor volumes during EGFR-TKI therapy in 24 patients in the pilot cohort with a total of 227 chest CT scans, and calculated tumor growth rate after the nadir in each patient with a graphical display. A representative case of volumetric tumor growth of in an EGFR-mutant NSCLC patient and the workflow is shown in Fig. 2. First, the user performed tumor volume measurement using the lung nodule analysis and segmentation tool in the Vitrea workstation (Fig. 2a), as described previously 8 . Then, the user saved the volume measurement results using "save to csv" option in the "Dictation Table" tab, which generates csv file of the measurements (Fig. 2a). The "snapshot" function with a camera icon (Fig. 2a) allowed the user save the graphical data of the segmentation and measurements. After performing the tumor volume measurements on all the scans of interest from the baseline to the most recent follow-up CT, the user can open the analytic module using the icon for "Longitudinal Analysis" on the workstation (Fig. 2b).

The analytic module opened in a separate window on the Vitrea workstation, and displayed a trend graph of the longitudinal changes of tumor volume in the lower half of the screen, initially using the absolute volume in $\mathrm{mm}^{3}$ as a default (Fig. 2b). Three sets of CT scans were displayed in the upper half of the screen, by default showing the baseline scan on the left, most recent prior study in the middle, and the current study on the right. The user can choose the scans for display, by manually dragging the white bar with blue arrowhead on the graph to the time point of interest, such as the nadir (Fig. 2b). The drop down menu allowed the user to choose the display mode of the graph, from the absolute volume to a "ratio" 
mode comparing with the baseline (indicating the baseline volume as "1.0 (100\%)") (Fig. $2 \mathrm{c})$, and a "log" mode using the natural logarithm of the actual volume $\left(\log _{\mathrm{e}} \mathrm{V}\right)(\mathrm{Fig} .2 \mathrm{~d})$. When the user selected the log mode, the module automatically and instantly calculated the tumor growth rate by fitting the line of best fit for all volume measurements from the nadir to the current scan, using the least square method. The tumor growth rate was displayed as the slope of the line, R, on the graph (Fig. 2d), which indicated the tumor growth rate for $\log _{\mathrm{e}} \mathrm{V}$ per month.

The rate can be interpreted using the previously published value, $0.15 / \mathrm{month}$, for the upper threshold to define slow tumor growth, to provide objective guide for therapeutic decision making at the interpretation of the current scan. For example, the case shown in Fig. 2 with the tumor growth rate of 0.040 /month and represents slow tumor growth, as the rate was less than $0.15 /$ month, indicating that the patient can remain on therapy and the clinician elected to keep the patient on therapy with erlotinib. In contrast, the example shown in Fig. 3a with the tumor growth rate of $0.207 /$ month, which was above $0.15 /$ month threshold and thus represented fast tumor growth, indicating it may be time to consider alternate or additional therapy.

Based on the input from the users, the module also allowed the selection of the time point, other than the nadir, from which the tumor growth rate was calculated. For this function, the user can manually drag the orange dotted line indicating the nadir to the time point of their interest, to see how the tumor volume is increasing during the period of their interest. For example, the user can drag the dotted line from the nadir to a more recent time point in the past few months, to evaluate the tumor growth rate focusing on the more recent time period (Fig. 3b).

\section{Inter and intraobserver variability of tumor growth rate evaluations}

The tumor volume growth rates measured by two readers in 24 patients in the pilot cohort showed high interobserver agreement with CCC of 0.9323 (95\%CI: 0.8477 - 0.9706) (Fig. 4a). Between two measurements by the first reader, the intraobserver agreement was also high, with CCC of 0.9668 (95\%CI: 0.9237 - 0.9858) (Fig. 4b). Using the 0.15/month threshold, the interpretation of the slow vs. fast tumor growth showed a perfect agreement for interobserver assessments $(\kappa=1.00)$, with all 24 patients having concordant interpretations. Intraobserver agreements were excellent ( $\kappa=0.895$ ), with only one of the 24 patients having different interpretations of slow growth versus rapid growth (Fig. $4 b$ ).

\section{DISCUSSION}

The present study described the development of an automated analytic module for tumor growth rate analysis, and demonstrated the functionality of the module in a pilot cohort of EGFR-mutant NSCLC patients treated with EGFR-TKI. High inter and intraobserver agreements were noted for growth rate assessments and interpretation, indicating the reliability of the approach for treatment decision guides during precision cancer therapy. Although the previous literature investigated tumor growth rate assessments as response and prognostic markers advanced cancers $27,28,31-33$, the development of automated module dedicated for tumor growth rate calculation for precision cancer therapy has not been 
previously described. In addition, though the reproducibility of tumor volume measurement on CT at one time point has been well demonstrated ${ }^{7-10}$, the reproducibility of volumetric tumor growth rate assessments using serial $\mathrm{CT}$ scans utilizing all time points during therapy has not been previously investigated.

The module utilized an already existing commercially available, FDA-cleared imageprocessing workstation equipped with tumor volume measurement tool that has been previously validated in advanced NSCLC patients. ${ }^{8}$ The strategy was chosen because of an advantage to ensure the smooth delivery of the module for clinical translation beyond our institution, which is a major long-term goal of the overall project. Additionally, the module is structured so that it can be added on to the existing workstations, with a scope for future modifications that will allow the module to be added and operate on other DICOM-based imaging processing workstations from different vendors for a broader use. The module also accepts DICOM-based images from any CT scanners regardless of the vendors, which is an essential feature for universal use. Given a large number of patients with advanced NSCLC diagnosed every year worldwide and an increasing use of precision therapy in oncology, the future direction toward universal application is essential for this type of technology.

The need for more automated analyses of tumor growth rate has been identified as an important issue in precision oncology, based on the clinical experiences in management of advanced cancer patients. The need for reproducible means for assessing slow progression had already been identified and the subject of prospective clinical trials for patients with EGFR-mutant NSCLC ${ }^{20}$. Therefore, the methods of growth rate calculations and threshold values to define slow progression are needed and we had generated a study plan based on the prior investigations in EGFR-mutant NSCLC patients. ${ }^{30,33}$ The graphical display and detailed functions were developed and optimized via collaborative interactions between the clinical team representing potential users and the engineering team. The close communications and interactive relationships were indispensable to develop the module that provides a technical solution for a specific cancer-related problem in the clinical setting, which proposes a paradigm for clinical translations of image-related innovations in oncology that can ultimately be deployed in the clinic.

Tumor growth rates after the nadir volume during EGFR-TKI therapy using the module demonstrated high inter and intraobserver agreements. Of note, the interpretation of the slow vs. fast tumor growth using the $0.15 /$ month threshold had a flawless interobserver agreement $(\kappa=1.00)$, indicating the reliability of the approach as a treatment decision guide between these two radiologists. Although high reproducibility of tumor volume measurements has been consistently shown in lung cancer and other tumors on $\mathrm{CT}$ at a single time point, ${ }^{7-10}$ the current study demonstrated high intra and interobserver agreements of tumor growth rate calculation using serial CT scans at all time points during therapy in each patient, indicating that volumetric growth rate can be an objective and reliable marker for therapeutic guidance in advanced NSCLC cancer patients treated with precision therapy. ${ }^{28,30,33}$

While the present study focused on EGFR-mutant NSCLC patients treated with EGFR-TKI as a well-studied cohort for the first step of module development, the analytic module for tumor growth rate has a wider applicability in other cohorts of lung cancer treated with 
effective targeted therapy directed against ALK, ROS1, and BRAF inhibitors. During therapy using these targeted agents, similar tumor volume dynamics with initial marked response and gradual tumor growth due to acquired resistance already have been noted, indicating tumor regrowth after nadir is a common issue in precision cancer therapy. Furthermore, recent investigations described longitudinal tumor burden dynamics as an important marker for treatment benefit and survival in patients treated with immunecheckpoint inhibitors, which suggests an additional area of oncology where the module can contribute to guide therapeutic decisions. Further testing of the module in different cohorts of advanced NSCLC patients treated with precision therapy and immunotherapy is ongoing.

The limitations of the study include a single institution experience with a small pilot cohort of patients assessed retrospectively. The threshold for slow tumor growth of $0.15 / \mathrm{month}$ has been previously reproduced; however, remains to be tested for an association with improved survival. Further studies in larger cohorts of patients with different TKIs are planned to validate the threshold for the association with survival, and to test the module in various clinical scenarios. Tumor volume measurement and growth rate assessment were performed using one dominant lung lesion per patients, rather than incorporating all lesions throughout the body. This method was used because our previous studies have shown that the tumor volume analysis of one dominant lesion can predict outcome in EGFR-mutant NSCLC patients treated with EGFR-TKI ${ }^{33,35}$. In addition, not all lesions may be suited for CT segmentation and volume measurements, leading to limitations and barriers such as lower reproducibility, increased time and efforts for measurements, and difficulty of standardization of the techniques. The tumor growth rate analysis focusing on one dominant lung lesion does not incorporate the rest of the systemic tumor burden into consideration in terms of therapeutic response and treatment guidance. Therefore, tumor volume analysis should be used in parallel with RECIST-based assessment which remains as a simple and practical measure of systemic tumor burden ${ }^{5,33,35}$, and provide an added value to RECISTbased evaluations in terms of treatment decisions especially when tumors are slowly progressing. The current study included evaluation of the module by two observers on Vitrea work station as an initial step. Future plans include prospective validation of the approach, as well as testing and validation in larger cohorts of patients at external sites involving a larger number of observers with different levels of experiences and using other DICOM-based workstation, to achieve the long-term goal for bringing the technology solution into the clinical settings, for which the current study serves as an important initial step.

In conclusion, the present study described the development of an automated analytic module for tumor growth rate in lung cancer patients treated with precision therapy, and demonstrated the functionality and reproducibility of the approach in a pilot cohort of advanced NSCLC patients with EGFR mutation. The initial results serve as a basis for further progress and application of the approach as a translational technology solution to guide cancer treatment in the era of precision oncology.

\section{ACKNOWLEDGEMENT}

Funding: This work was supported by the National Institutes of Health R01CA203636 (NCI). 


\section{REFEENCES}

1. Miller AB, Hoogstraten B, Staquet M, et al.: Reporting results of cancer treatment. Cancer 47:20714, 1981 [PubMed: 7459811]

2. Therasse P, Arbuck SG, Eisenhauer EA, et al.: New guidelines to evaluate the response to treatment in solid tumors. European Organization for Research and Treatment of Cancer, National Cancer Institute of the United States, National Cancer Institute of Canada. J Natl Cancer Inst 92:205-16, 2000 [PubMed: 10655437]

3. Eisenhauer EA, Therasse P, Bogaerts J, et al.: New response evaluation criteria in solid tumours: revised RECIST guideline (version 1.1). Eur J Cancer 45:228-47, 2009 [PubMed: 19097774]

4. Nishino M, Jagannathan JP, Ramaiya NH, et al.: Revised RECIST guideline version 1.1: What oncologists want to know and what radiologists need to know. AJR Am J Roentgenol 195:281-9, 2010 [PubMed: 20651182]

5. Nishino M, Jagannathan JP, Krajewski KM, et al.: Personalized tumor response assessment in the era of molecular medicine: cancer-specific and therapy-specific response criteria to complement pitfalls of RECIST. AJR Am J Roentgenol 198:737-45, 2012 [PubMed: 22451534]

6. Nishino M, Cardarella S, Dahlberg SE, et al.: Radiographic assessment and therapeutic decisions at RECIST progression in EGFR-mutant NSCLC treated with EGFR tyrosine kinase inhibitors. Lung Cancer 79:283-8, 2013 [PubMed: 23254265]

7. Zhao B, James LP, Moskowitz CS, et al.: Evaluating variability in tumor measurements from sameday repeat CT scans of patients with non-small cell lung cancer. Radiology 252:263-72, 2009 [PubMed: 19561260]

8. Nishino M, Guo M, Jackman DM, et al.: CT tumor volume measurement in advanced non-small-cell lung cancer: Performance characteristics of an emerging clinical tool. Acad Radiol 18:54-62, 2011 [PubMed: 21036632]

9. Mozley PD, Bendtsen C, Zhao B, et al.: Measurement of tumor volumes improves RECIST-based response assessments in advanced lung cancer. Transl Oncol 5:19-25, 2012 [PubMed: 22348172]

10. Nishino M, Hatabu H, Johnson BE, et al.: State of the art: Response assessment in lung cancer in the era of genomic medicine. Radiology 271:6-27, 2014 [PubMed: 24661292]

11. Kris MG, Johnson BE, Berry LD, et al.: Using multiplexed assays of oncogenic drivers in lung cancers to select targeted drugs. JAMA 311:1998-2006, 2014 [PubMed: 24846037]

12. Zhou C, Wu YL, Chen G, et al.: Erlotinib versus chemotherapy as first-line treatment for patients with advanced EGFR mutation-positive non-small-cell lung cancer (OPTIMAL, CTONG-0802): a multicentre, open-label, randomised, phase 3 study. Lancet Oncol 12:735-42, 2011 [PubMed: 21783417]

13. Sequist LV, Martins RG, Spigel D, et al.: First-line gefitinib in patients with advanced non-smallcell lung cancer harboring somatic EGFR mutations. J Clin Oncol 26:2442-9, 2008 [PubMed: 18458038]

14. Rosell R, Carcereny E, Gervais R, et al.: Erlotinib versus standard chemotherapy as first-line treatment for European patients with advanced EGFR mutation-positive non-small-cell lung cancer (EURTAC): a multicentre, open-label, randomised phase 3 trial. Lancet Oncol 13:239-46, 2012 [PubMed: 22285168]

15. Mok TS, Wu YL, Thongprasert S, et al.: Gefitinib or carboplatin-paclitaxel in pulmonary adenocarcinoma. N Engl J Med 361:947-57, 2009 [PubMed: 19692680]

16. Maemondo M, Inoue A, Kobayashi K, et al.: Gefitinib or chemotherapy for non-small-cell lung cancer with mutated EGFR. N Engl J Med 362:2380-8, 2010 [PubMed: 20573926]

17. Soria JC, Ohe Y, Vansteenkiste J, et al.: Osimertinib in Untreated EGFR-Mutated Advanced NonSmall-Cell Lung Cancer. N Engl J Med 378:113-125, 2018 [PubMed: 29151359]

18. Nishino M, Jackman DM, Hatabu H, et al.: Imaging of lung cancer in the era of molecular medicine. Acad Radiol 18:424-36, 2011 [PubMed: 21277232]

19. Sequist LV, Waltman BA, Dias-Santagata D, et al.: Genotypic and histological evolution of lung cancers acquiring resistance to EGFR inhibitors. Sci Transl Med 3:75ra26, 2011

20. Park K, Yu CJ, Kim SW, et al.: First-Line Erlotinib Therapy Until and Beyond Response Evaluation Criteria in Solid Tumors Progression in Asian Patients With Epidermal Growth Factor 
Receptor Mutation-Positive Non-Small-Cell Lung Cancer: The ASPIRATION Study. JAMA Oncol 2:305-12, 2016 [PubMed: 26720423]

21. Riely GJ, Kris MG, Zhao B, et al.: Prospective assessment of discontinuation and reinitiation of erlotinib or gefitinib in patients with acquired resistance to erlotinib or gefitinib followed by the addition of everolimus. Clin Cancer Res 13:5150-5, 2007 [PubMed: 17785570]

22. Camidge DR, Bang YJ, Kwak EL, et al.: Activity and safety of crizotinib in patients with ALKpositive non-small-cell lung cancer: updated results from a phase 1 study. Lancet Oncol 13:10119, 2012 [PubMed: 22954507]

23. Tani T, Yasuda H, Hamamoto J, et al.: Activation of EGFR Bypass Signaling by TGFalpha Overexpression Induces Acquired Resistance to Alectinib in ALK-Translocated Lung Cancer Cells. Mol Cancer Ther, 2015

24. Kim TM, Song A, Kim DW, et al.: Mechanisms of Acquired Resistance to AZD9291: A MutationSelective, Irreversible EGFR Inhibitor. J Thorac Oncol 10:1736-44, 2015 [PubMed: 26473643]

25. Isozaki H, Ichihara E, Takigawa N, et al.: Non-small cell lung cancer cells acquire resistance to the ALK inhibitor alectinib by activating alternative receptor tyrosine kinases. Cancer Res, 2015

26. Eberlein CA, Stetson D, Markovets AA, et al.: Acquired Resistance to the Mutant-Selective EGFR Inhibitor AZD9291 Is Associated with Increased Dependence on RAS Signaling in Preclinical Models. Cancer Res 75:2489-500, 2015 [PubMed: 25870145]

27. Ferte C, Fernandez M, Hollebecque A, et al.: Tumor growth rate is an early indicator of antitumor drug activity in phase I clinical trials. Clin Cancer Res 20:246-52, 2014 [PubMed: 24240109]

28. Gomez-Roca C, Koscielny S, Ribrag V, et al.: Tumour growth rates and RECIST criteria in early drug development. Eur J Cancer 47:2512-6, 2011 [PubMed: 21763126]

29. Levy A, Hollebecque A, Ferte C, et al.: Tumor assessment criteria in phase I trials: beyond RECIST. J Clin Oncol 31:395, 2013 [PubMed: 23248247]

30. Nishino M, Dahlberg SE, Cardarella S, et al.: Volumetric tumor growth in advanced non-small cell lung cancer patients with EGFR mutations during EGFR-tyrosine kinase inhibitor therapy: developing criteria to continue therapy beyond RECIST progression. Cancer 119:3761-8, 2013 [PubMed: 23922022]

31. Stein WD, Gulley JL, Schlom J, et al.: Tumor regression and growth rates determined in five intramural NCI prostate cancer trials: the growth rate constant as an indicator of therapeutic efficacy. Clin Cancer Res 17:907-17, 2011 [PubMed: 21106727]

32. Stein WD, Wilkerson J, Kim ST, et al.: Analyzing the pivotal trial that compared sunitinib and IFN-alpha in renal cell carcinoma, using a method that assesses tumor regression and growth. Clin Cancer Res 18:2374-81, 2012 [PubMed: 22344231]

33. Nishino M, Dahlberg SE, Fulton LE, et al.: Volumetric Tumor Response and Progression in EGFRmutant NSCLC Patients Treated with Erlotinib or Gefitinib. Acad Radiol 23:329-36, 2016 [PubMed: 26776293]

34. Nishino M, Jackman DM, DiPiro PJ, et al.: Revisiting the relationship between tumour volume and diameter in advanced NSCLC patients: An exercise to maximize the utility of each measure to assess response to therapy. Clin Radiol 69:841-8, 2014 [PubMed: 24857677]

35. Nishino M, Dahlberg SE, Cardarella S, et al.: Tumor volume decrease at 8 weeks is associated with longer survival in EGFR-mutant advanced non-small-cell lung cancer patients treated with EGFR TKI. J Thorac Oncol 8:1059-68, 2013 [PubMed: 23787800]

36. Clunie DA: DICOM structured reporting and cancer clinical trials results. Cancer Inform 4:33-56, 2007 [PubMed: 19390663]

37. Demicheli R, Foroni R, Ingrosso A, et al.: An exponential-Gompertzian description of LoVo cell tumor growth from in vivo and in vitro data. Cancer Res 49:6543-6, 1989 [PubMed: 2819710]

38. Looney WB, Trefil JS, Hopkins HA, et al.: Solid tumor models for assessment of different treatment modalities: therapeutic strategy for sequential chemotherapy with radiotherapy. Proc Natl Acad Sci U S A 74:1983-7, 1977 [PubMed: 194245]

39. Looney WB, Trefil JS, Schaffner JC, et al.: Solid tumor models for the assessment of different treatment modalities: I. Radiation-induced changes in growth rate characteristics of a solid tumor model. Proc Natl Acad Sci U S A 72:2662-6, 1975 [PubMed: 170612] 
40. Looney WB, Trefil JS, Schaffner JG, et al.: Solid tumor models for the assessment of different treatment modalities: systematics of response to radiotherapy and chemotherapy. Proc Natl Acad Sci U S A 73:818-22, 1976 [PubMed: 176656] 


\section{Highlights}

- Analytic module for tumor growth rate is developed to guide precision cancer therapy

- Functionality and reproducibility is shown in EGFR-mutant NSCLC treated with TKI

- The approach may serve as a translational technology solution in precision oncology 


\section{Imaging processing workstation}

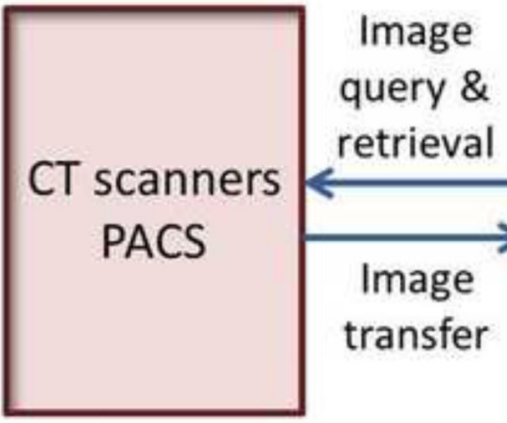

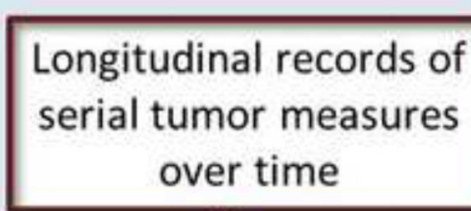

Trend graph of tumor volume dynamics $\downarrow$

Calculation of tumor volume growth rate after nadir

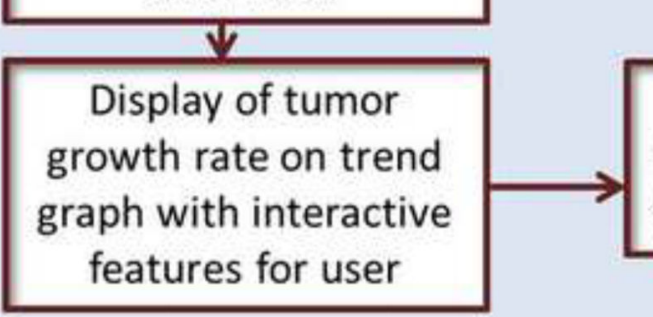

Interpret and use the results to guide treatment decisions

Fig. 1.

Workflow of serial tumor volume measurements and display, calculation and interpretation of tumor growth rate using the analytic module added to the image-processing workstation. 

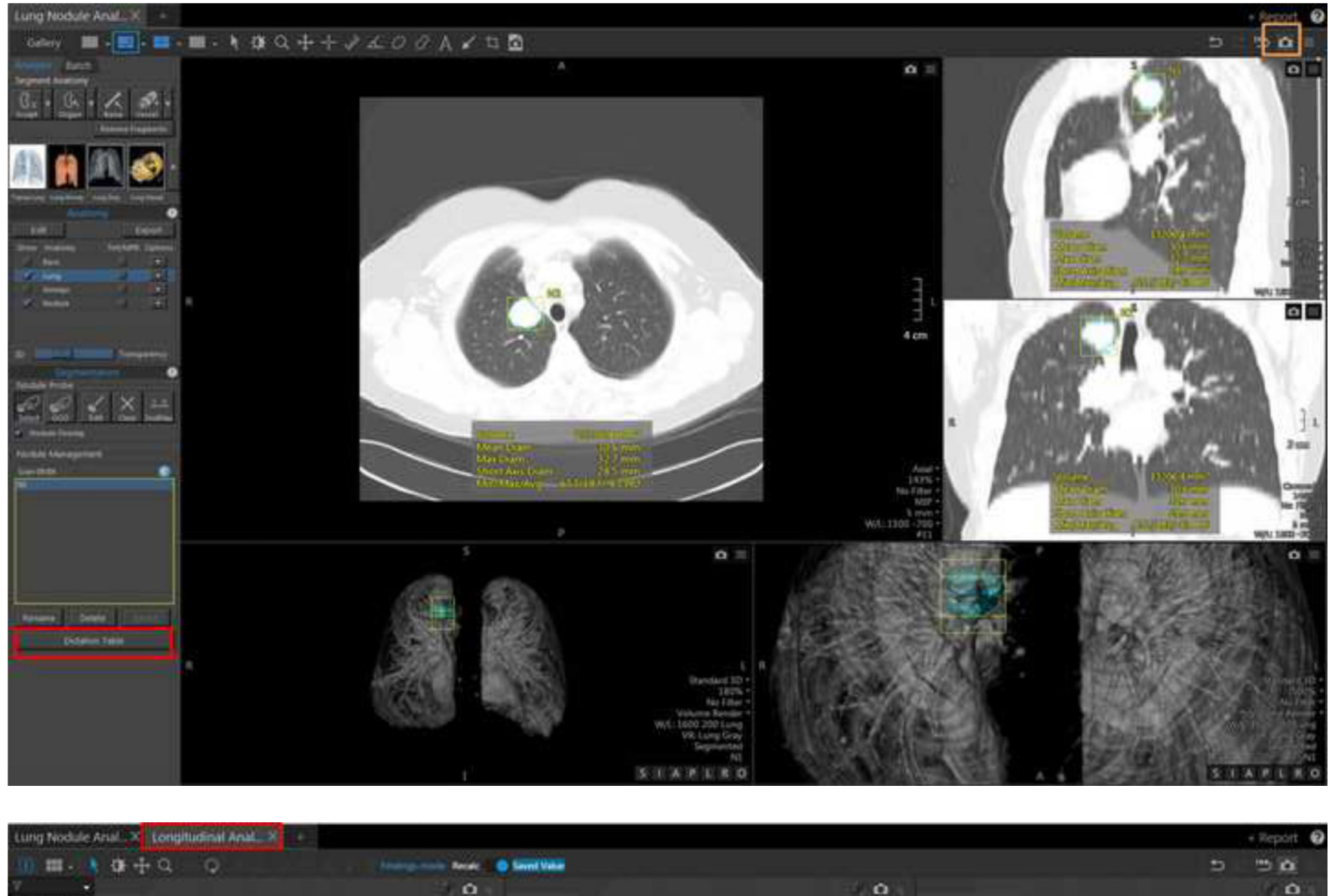

insucter
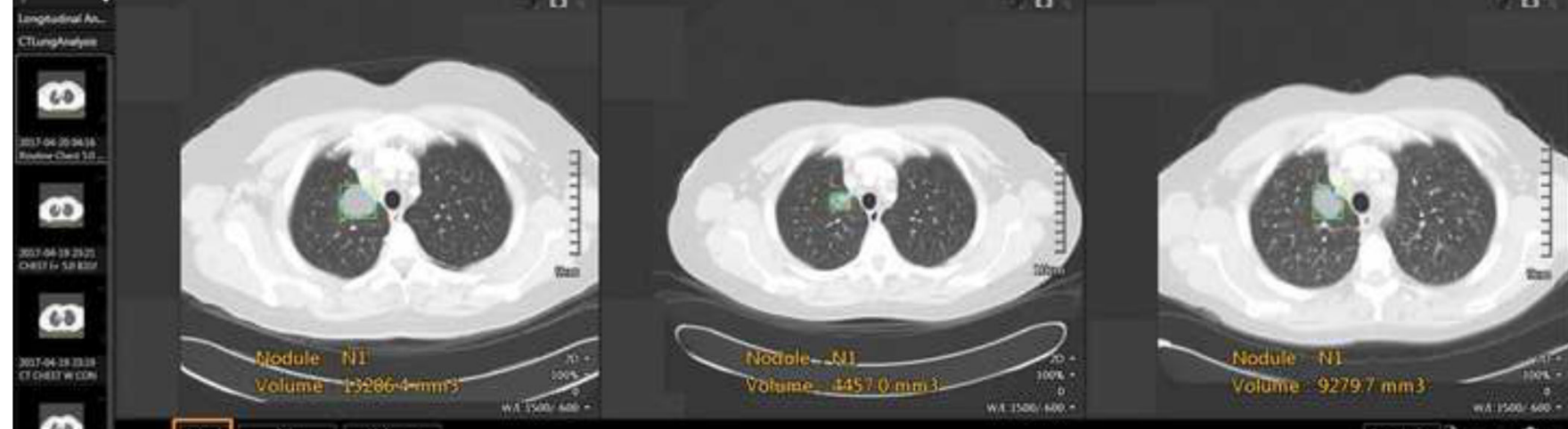

$\omega$
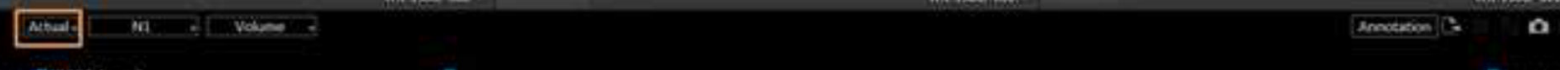

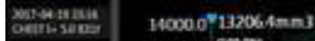

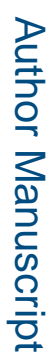

(C) 100000

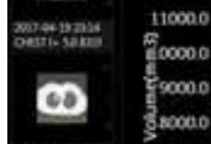

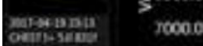

68

60000 | $56709 \mathrm{~mm}^{3}$

lew osermas

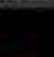

$a$

$+0000$

ind

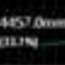

Noverom ressuma

12000

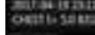

20. $\operatorname{stan} 2004$ A A whentsis

$n$

$30 \quad 40$

Sotschem
tand

inso tans 

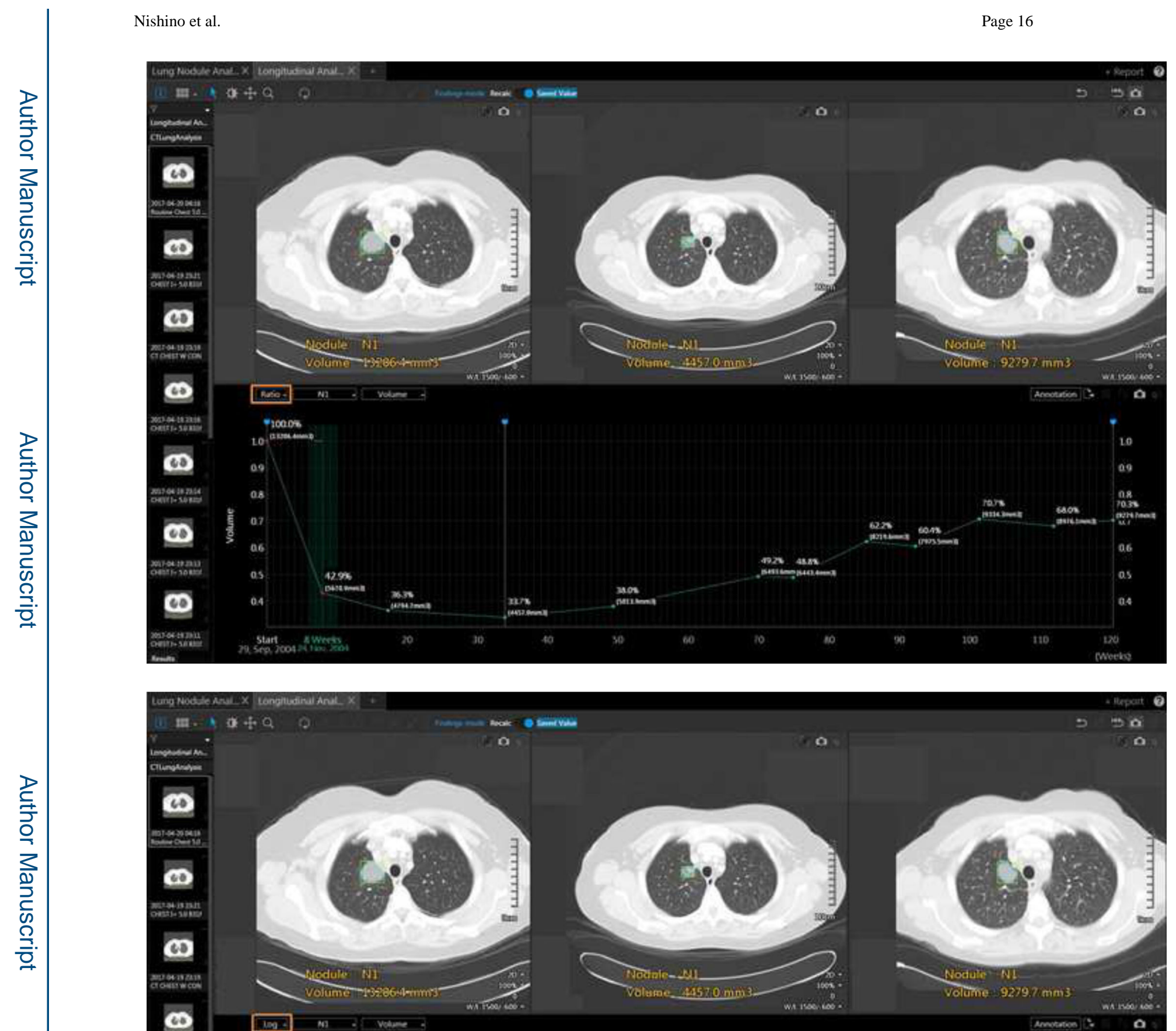

120\%

$\infty$

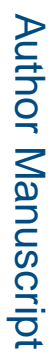

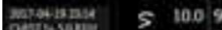

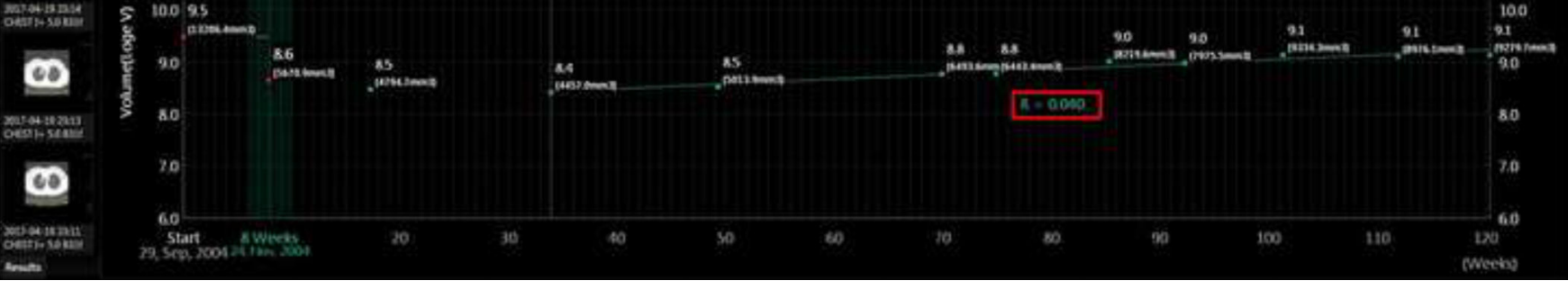

Fig. 2.

Eur J Radiol. Author manuscript; available in PMC 2019 December 01. 
A representative case of volumetric tumor growth analysis using the module in an EGFRmutant NSCLC patient, representing a slow tumor growth after the nadir. a. The user performs tumor volume measurement using the lung nodule analysis and segmentation tool in the workstation that has been previously validated. "Dictation Table" tab (red box) allows the user to save the volume measurement results using "save to csv" option. Using the "snapshot" function with a camera icon (orange box), the user can also save the graphical data of the segmentation and measurements.

b. Once the tumor volume measurements on all the scans of interest during therapy are performed and saved, the user opens the analytic module, "Longitudinal Analysis" on the workstation (red box) in a separate window on the workstation. The module displays a trend graph of the longitudinal tumor volume changes using the absolute volume in $\mathrm{mm}^{3}$, along with three CT scans with corresponding white vertical bars with blue arrowheads on the graph. The scans for display can be chosen by the user, by manually dragging the vertical bar on the graph to the time point of interest on the graph. (Green bar indicates 8-week time point from the initiation of therapy, with a range of $+/-2$ weeks as a green shaded area, which is a commonly used time point for the $1^{\text {st }}$ follow up CT in these patients and has been shown to predict subsequent prognosis.)

c. The user can change the display unit using the drop down menu, from the absolute volume to a "ratio" mode (orange box) for example, displaying the proportional changes of the volume in comparison with the baseline. The baseline volume is indicated as "1.0 (100\%)". d. When user chooses a "log" mode (orange box), the graph displays the volume changes using the natural $\log$ arithm of the actual volume $\left(\log _{\mathrm{e}} \mathrm{V}\right)$ originally measured in $\mathrm{mm}^{3}$. When this mode is chosen, the module automatically detects the nadir and calculate the tumor growth rate using all volume measurements from the nadir to the current scan. The tumor growth rate is displayed as the slope of the best-fitted line, $\mathrm{R}$, on the graph (red box), indicating the tumor growth rate for $\log _{\mathrm{e}} \mathrm{V}$ per month. The growth rate since the nadir is $0.040 /$ month, which is below the threshold of $0.15 /$ month. 

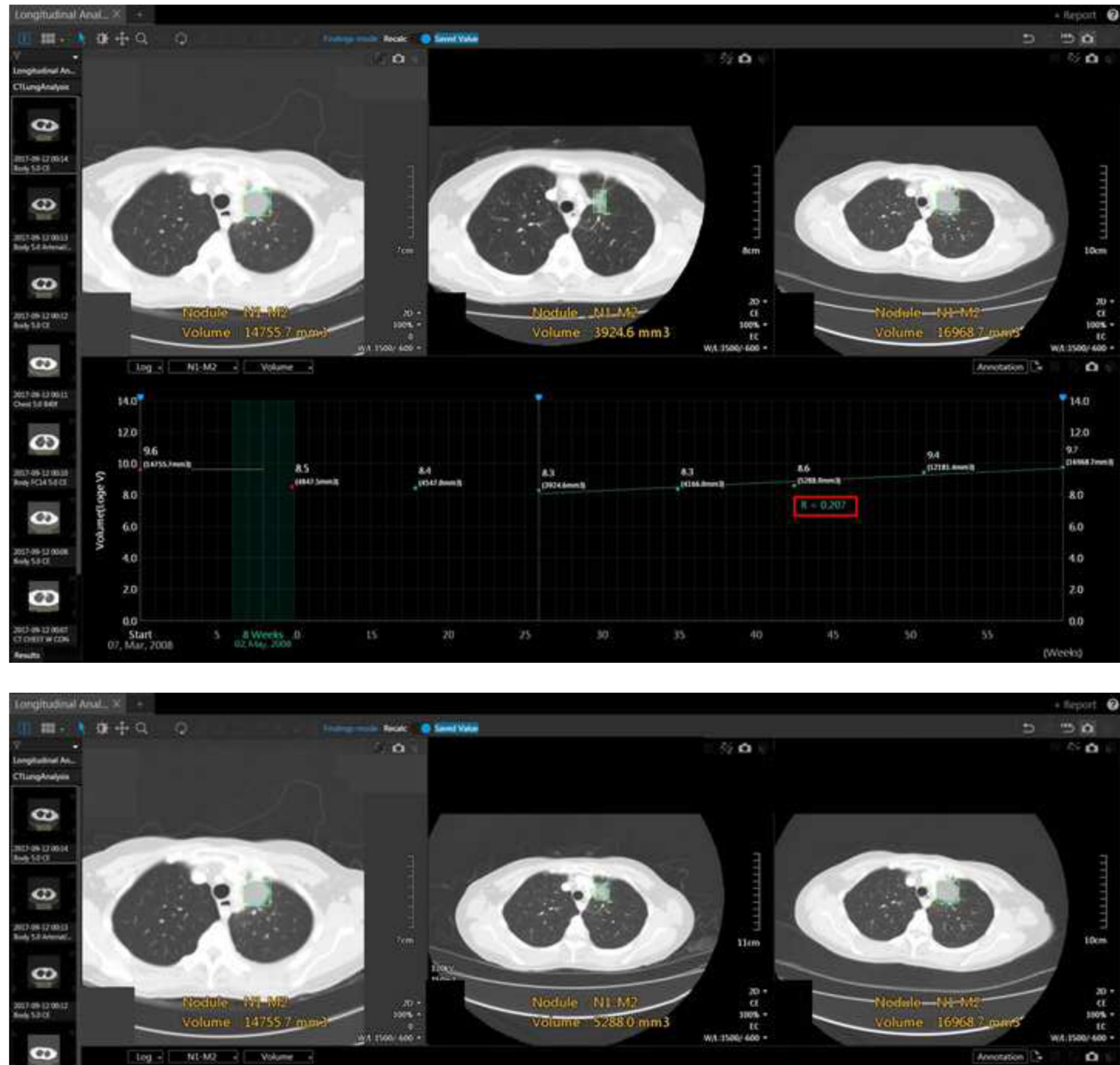

CD 200
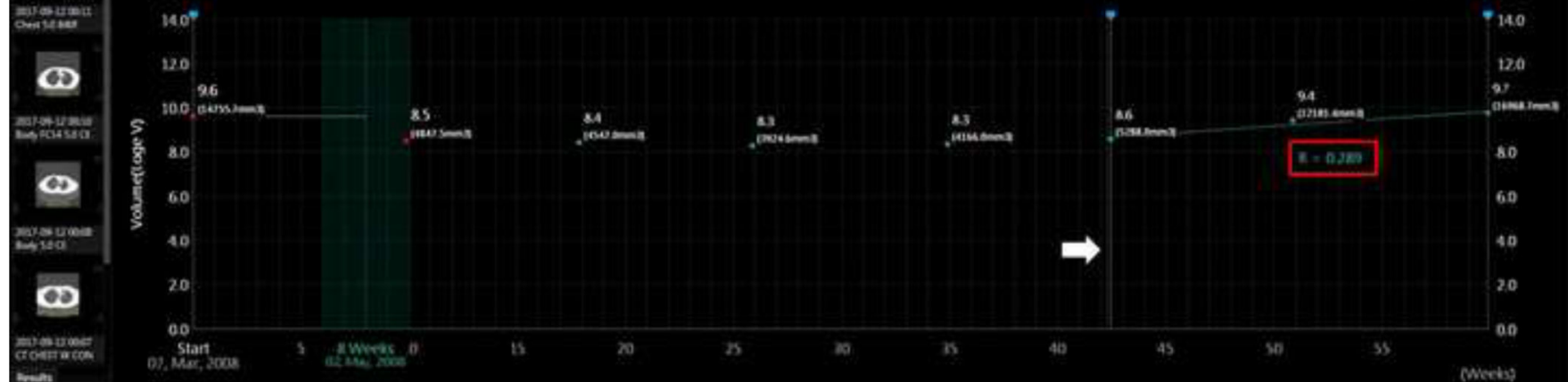

$\infty$

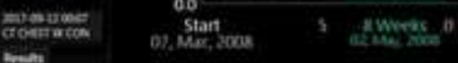

Fig. 3.

Eur J Radiol. Author manuscript; available in PMC 2019 December 01. 
A representative case of fast tumor growth after the nadir evaluated by the module. . Another case of EGFR-mutant NSCLC patient treated with EGFR-TKI shows the tumor growth rate after the nadir of $0.207 /$ month (red box), which is above $0.15 /$ month threshold, representing fast tumor growth.

b. When using the log mode for tumor growth calculation, the user can manually drag the orange dotted line (arrow) originally set to indicate the nadir, to the time point of their interest. For example, when the dotted line was moved from the nadir to a more recent time point in the past few months, the tumor growth rate during the more recent period defined by the user can be obtained (red box). 


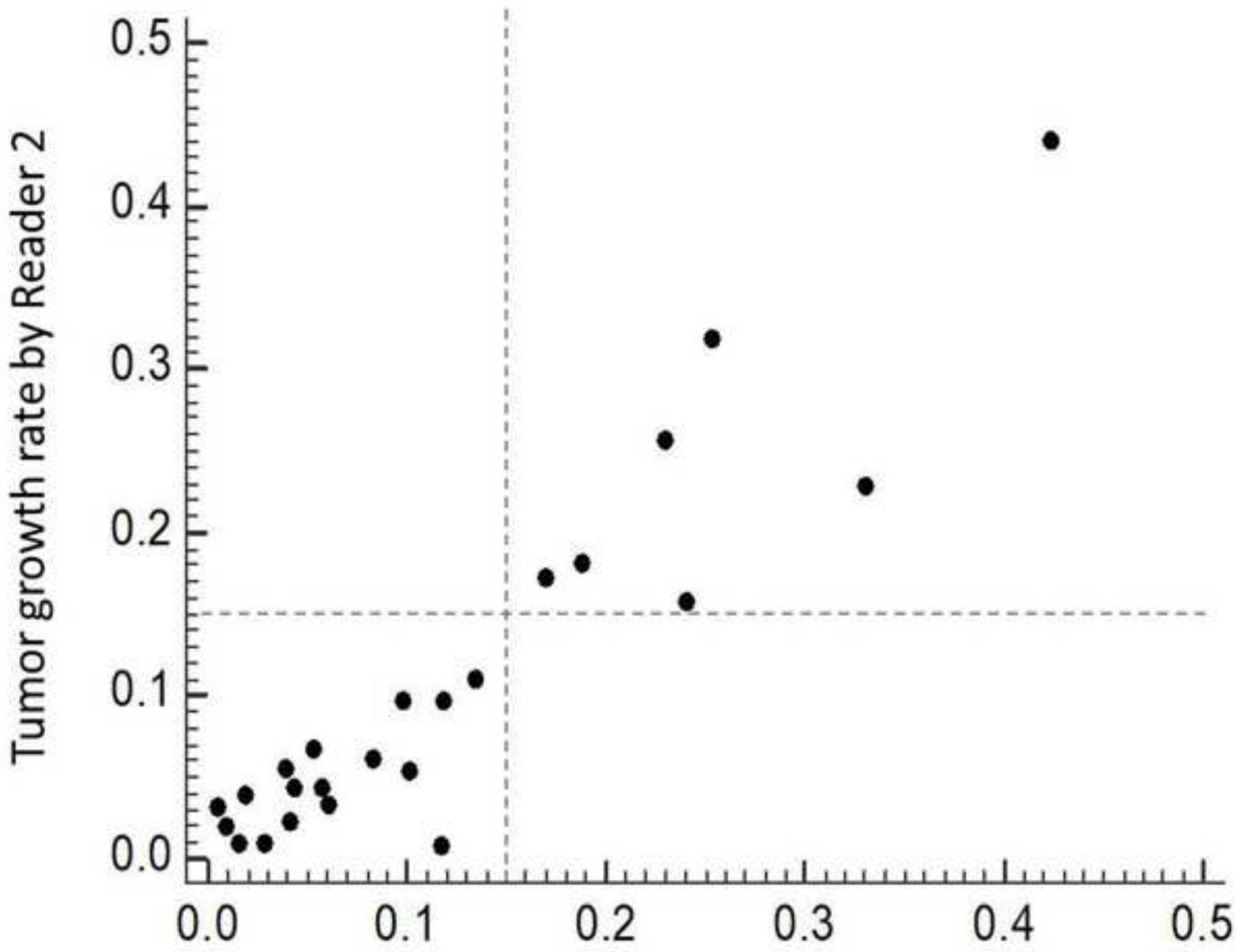

Tumor growth rate by Reader 1 


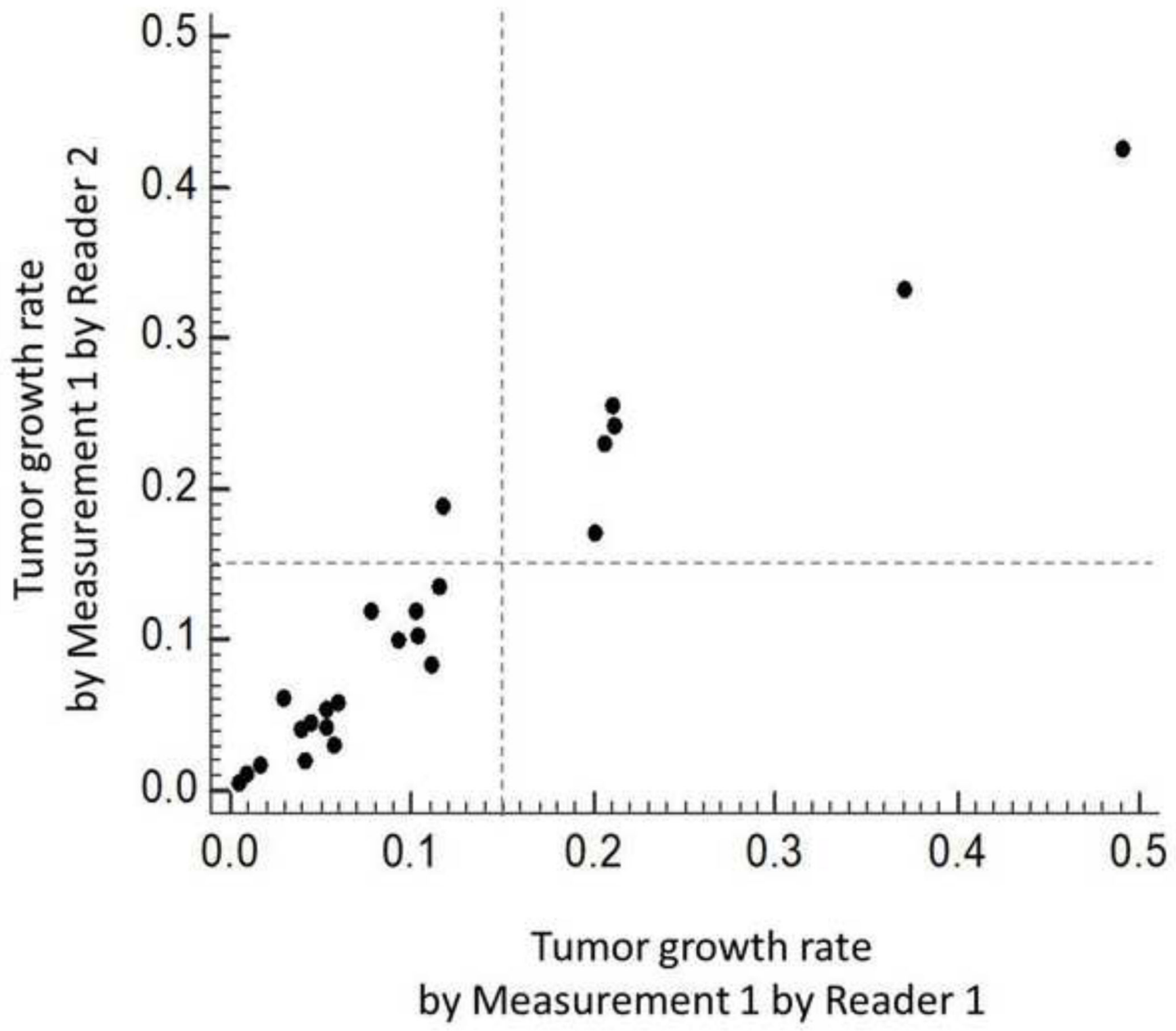

Fig. 4.

Inter (a) and intraobserver (b) variability of tumor growth rate per month, measured using natural $\log$ arithm of the actual volume $\left(\log _{\mathrm{e}} \mathrm{V}\right)$ originally measured in $\mathrm{mm}^{3}$. High inter and intraobserver agreements were noted for tumor growth rates, with concordance correlation coefficients of 0.9323 (a) and 0.9668 (b), respectively. Dotted lines represent the previously identified threshold of $₫ \mathbf{5} .15 /$ month. Interpretation of slow versus fast tumor growth using the threshold had a perfect interobserver agreement $(\kappa=1.00)(\mathrm{a})$, and an almost perfect intraobserver agreement $(\kappa=0.895)(b)$. 